Talebi, S., Koskela, L., and Tzortzopoulos, P. (2018). "Tolerance Compliance Measurement Using Terrestrial Laser Scanner." In: Proc. 26 th Annual Conference of the International. Group for Lean Construction (IGLC), González, V.A. (ed.), Chennai, India, pp. 166-176. DOI: doi.org/10.24928/2018/0539. Available at: www.iglc.net.

\title{
TOLERANCE COMPLIANCE MEASUREMENT USING TERRESTRIAL LASER SCANNER
}

\author{
Saeed Talebi ${ }^{1}$, Lauri Koskela ${ }^{2}$, Patricia Tzortzopoulos ${ }^{3}$
}

\begin{abstract}
Terrestrial laser scanning (TLS) provides remote sensing and a quick and comprehensive technique for deviation analyses. Its application for precision surveying purposes is becoming a common practice. There are many interdependent parameters that determine whether the accuracy obtained during the data collection and registration is appropriate to perform deviation analyses. Also, the accuracy of deviation analyses is reflected on visualisation/demonstration of results. However, the focus of previous research works has often been on either data acquisition, data registration, deviation analyses, or visualisation of results. The research described in this paper consolidates and formalises the existing methods in the literature and practice for data acquisition and data processing. In doing so, the aim is to develop a holistic method termed Tolerance Compliance Measurement (TCM) using TLS to obtain results from deviation analyses with the desired accuracy. Moreover, unlike the previous research works that mainly focus on assessment of flatness of surfaces, the appropriateness of the most common algorithms for assessment of different types of geometric variation is explored. The results show that the application of TLS and commercially available software are versatile although not complete for analyses of different types of geometric variation.
\end{abstract}

\section{KEYWORDS}

Laser scanning, deviation analyses, data acquisition, data registration, visualisation of deviation maps, demonstration of deformation analyses, geometric variations, enabling Lean with IT.

1 Lecturer and PhD Researcher, School of Art, Design and Architecture, University of Huddersfield, Queen Street Building, Queen St, Huddersfield, West Yorkshire, HD1 3DU, United Kingdom.

Saeed.Talebi@hud.ac.uk

2 Professor, School of Art, Design and Architecture, University of Huddersfield, Queen Street Building, Queen St, Huddersfield, West Yorkshire, HD1 3DU, United Kingdom. L.Koskela@ hud.ac.uk

3 Professor, School of Art, Design and Architecture, University of Huddersfield, Queen Street Building, Queen St, Huddersfield, West Yorkshire, HD1 3DU, United Kingdom. P.Tzortzopoulos@ hud.ac.uk 


\section{INTRODUCTION}

Fixing defects associated with tolerances is time consuming, costly and onerous (Milberg and Tommelein 2005). In spite of increasing calls for waste reduction and an improved quality of buildings, Forcada et al. (2016) estimate that tolerance-related defects are amongst the most common and recurring defects in construction projects and make up more than 9 percent of the overall number of defects. One of the factors that can help minimise defects is to improve inspection methods used by surveyors and engineers (Yates and Lockley 2002). More specifically, defects associated with tolerances, called tolerance problems hereafter, can be mitigated by changing the inspection techniques and gaining better control of the magnitude of dimensional and geometric variations (Landin 2010). Conventional inspection methods use sampling techniques (Phares et al. 2004), some of them are limited to the need for surface contact (Bosché and Guenet 2014), and they depend on inspectors' subjective assessments (Anil et al. 2011). However, the use of conventional inspection methods has remained time-consuming, laborious, and therefore ineffective, although some of them are relatively accurate (Phares et al. 2004). As a result, such methods often cannot identify tolerance problems early and comprehensively during the construction process (Akinci et al. 2006) and results obtained from them may not be reliable (Phares et al. 2004). For instance, when assessing the flatness of concrete slabs by using the total station, only a few points representing the whole surface are collected. The elevations of the collected points are measured to determine their vertical deviations from the nominal elevation (Tang et al. 2011). Such method gives an incomplete and sometimes incorrect understanding of the achieved flatness to surveyors because surfaces that have higher deviations than permissible limits may not be controlled (Bosché and Guenet 2014). Communication of surveying results obtained from conventional methods is another problem area as inspectors may have different approaches to report the results (Anil et al. 2013). The lack of an effective standard method for communication may result in misinterpretation among project participants (Phares et al. 2004).

The terrestrial laser scanner (TLS) has been proven to be useful for a variety of applications including deviation analyses. Various methods for: (a) data acquisition (e.g., Wilkes et al. 2017), (b) registration (e.g., Olsen et al. 2009), (c) deviation analyses (e.g., Holst and Kuhlmann 2016), and (d) visualisation/demonstration of deviation analyses (e.g., Anil et al. 2013) have been proposed. However, a review of the literature reveals that there is not any current research work that proposes a holistic process consolidating these four independent fields of research for measuring geometric variations. To improve the accuracy of data registration and data analyses, and to improve the interoperability of results, it is suggested to have a formal process specifically for measuring geometric variations using TLS. Such a process must be holistic, that is it should start from data acquisition and extend to visualisation/demonstration of analyses. This is because the accuracy of deviation analyses depends on the way data is collected and registered; the accuracy of deviation analyses is also reflected in visualisation/demonstration. Hence, it is not a sufficient practice to consider these steps independently, especially when using them to measure variations that require a high level of accuracy. Moreover, most of the existing research works in this realm of research are about the assessment of surface 
flatness, whereas the capability of existing commercially available software for deviation analyses can also be used to assess other types of geometric variations (Nahangi and Haas 2014). Here the question arises: What method of deviation analysis is most suitable for each type of tolerances? This question can be addressed if types of tolerances are welldefined and they are associated with different methods of deviation analyses.

The topic of tolerances should be investigated from the lean construction perspective (Milberg and Tommelein 2005). This research employs one of the foundational elements of lean which is process standardisation. The standardisation of the best-known practice helps to maintain a regular timing and output of the process (Liker 2004), and to continually improve the design of that process (Womack and Jones 1997). This paper is a first attempt to propose a standardised process termed TCM using TLS: (a) to provide practical recommendations for capturing 3D data sets, thereby facilitating registration of data sets, (b) to propose a minimum viable workflow for data registration by which a high level of accuracy can be obtained, (c) to explore appropriateness of common methods of deviation analyses available for each type of tolerances, and (d) to explore effective methods for visualisation/demonstration of the results.

\section{LITERATURE REVIEW}

\section{DATA ACQUISITION AND REGISTRATION}

Two main parameters that impact the accuracy of data sets during data acquisition are: (a) distance, and (b) resolution setting (Kim et al. 2014). Scans with common targets and data should be aligned and merged to create a complete image of the scanning domain and to achieve registration with the desired accuracy (Olsen et al. 2009). Scans can be aligned together by applying either direct or indirect georeferencing methods. In direct georeferencing methods, targets with known coordinates are used. Coordinates can be obtained through the total station. In indirect georeferencing, software aligns the scans based on common data in neighbouring scans (Olsen et al. 2009). The final registered data is a set of points with known X, Y, and Z coordinates (Kim et al. 2014).

\section{DEVIATION ANALYSES}

The most common algorithm to measure deviations on surfaces is as follows: (a) a reference plane is set up, (b) data noises are smoothened, (c) the deviation between the points acquired and the reference plane is computed, and (d) surface regions, where their deviations are larger than the threshold specified by the user, are detected (Tang et al. 2010). In this algorithm, the reference plane is set up by fitting the best primitive shapes or triangulating the point cloud data set (Olsen et al. 2009). One type of deviation analysis is deformation analysis of the structural members. The deformation analysis can be performed in two ways: (a) the deformation in the surface of an object is determined from a given reference plane. The position and orientation of the reference plane can be either based on the nominal parameters in design, or its position and orientation are estimated as part of the deformation analysis; (b) the surface of an object is scanned twice or more times. The deformation is determined by computing the deviation between the 
position and orientation of surfaces at different points in time. The reference surface in this scenario is defined by the first scan (Holst and Kuhlmann 2016).

\section{VISUALISATION OF DEVIATION ANALYSES}

Once deviations were computed, they can be visualised in several ways by generating a colour map. There are two common categories of colour maps: continuous and binary colour maps (Anil et al. 2011). The focus of this paper is on the continuous colour map. In this type of visualisation, a colour to every deviation value is assigned according to a gradient colouring range (Anil et al. 2013). The continuous colour map itself can be either signed or unsigned. This paper utilises the former method, in which different colours are assigned to distinguish between positive and negative deviations (Anil et al. 2011). The reason behind these choices is that the authors believe they are more effective in practice.

\section{DIFFERENT TYPES OF TOLERANCES}

Talebi et al. (submitted) propose a method termed Geometric Dimensioning and Tolerancing in Construction (GD\&TIC). The ultimate goal of this method is to develop a common language to facilitate the communication of tolerance information throughout the design, construction and inspection process. GD\&TIC specifies the permitted variations in size, form, orientation and location of features on a component. Also, it consists of a total of six characteristics that represent the types of tolerance (Table 1).

Table 1: Tolerance types, their characteristics, and their applications

(Talebi et al. submitted)

\begin{tabular}{|c|c|c|}
\hline Type of Tolerance & Characteristics & Applications \\
\hline \multirow[t]{2}{*}{$\begin{array}{l}\text { Form: It establishes the } \\
\text { shape of a surface. }\end{array}$} & $\begin{array}{l}\text { Straightness: It represents how straight a } \\
\text { surface is on a feature along a line. }\end{array}$ & $\begin{array}{l}\text { It is used to control the beams and } \\
\text { columns that are prone to deformation. }\end{array}$ \\
\hline & $\begin{array}{l}\text { Flatness: It demonstrates the amount of } \\
\text { deviation of flatness that a surface is allowed } \\
\text { to have. }\end{array}$ & It controls the flatness of a floor slab. \\
\hline \multirow[t]{2}{*}{$\begin{array}{l}\text { Orientation: It describes the } \\
\text { relationship between } \\
\text { features and datums at } \\
\text { particular angles. }\end{array}$} & $\begin{array}{l}\text { Perpendicularity: It is a condition used to } \\
\text { ensure that a surface centre plane, or axis is } \\
\text { exactly at a right angle relative to a reference } \\
\text { plane. }\end{array}$ & $\begin{array}{l}\text { The Perpendicularity Control should } \\
\text { mainly be used for components for } \\
\text { which plumbness tolerances are a major } \\
\text { concern. }\end{array}$ \\
\hline & $\begin{array}{l}\text { Parallelism: It limits the amount of variation } \\
\text { allowed over an entire plane, from being } \\
\text { parallel to the reference plane. }\end{array}$ & $\begin{array}{l}\text { When two surfaces should maintain } \\
\text { constant distance, the Parallelism } \\
\text { Control is used. }\end{array}$ \\
\hline $\begin{array}{l}\text { Location: It establishes the } \\
\text { position of the feature } \\
\text { relative to a datum. }\end{array}$ & $\begin{array}{l}\text { Position: It is the location tolerance of a } \\
\text { feature relative to its nominal position. }\end{array}$ & $\begin{array}{l}\text { The Position Control is mainly used for } \\
\text { three purposes: (a) to control the } \\
\text { location of components such as } \\
\text { columns and beams, (b) to control the } \\
\text { distance between those components, } \\
\text { and (c) to control the coaxiality between } \\
\text { those components. }\end{array}$ \\
\hline $\begin{array}{l}\text { Profile: It is the outline of a } \\
\text { part feature and the True } \\
\text { Profile is the exact profile of } \\
\text { part feature. }\end{array}$ & $\begin{array}{l}\text { Profile of a surface: The Surface Profile } \\
\text { Control limits the amount of variation that the } \\
\text { surface of a feature can have in relation to its } \\
\text { True Profile. }\end{array}$ & $\begin{array}{l}\text { It is primarily used to control the level of } \\
\text { surfaces }\end{array}$ \\
\hline
\end{tabular}




\section{METHODOLOGY}

The paper consists of a review of the literature and collection of empirical data. Previous studies in the realm of data acquisition using TLS, data registration, deviation analyses and visualisation were investigated. The field researcher (lead author) observed the practice of a firm delivering 3D laser scanning services for two days and the practice of a software vendor company making applications for automated deviation analyses for three days. The aim of these observations was to understand relatively advanced practices from data acquisition to visualisation of deviation analyses in the industry. Also, the empirical data was collected by scanning a building and a warehouse. All scans were acquired by FARO Focus 3D X130. The 3D data sets were registered using the FARO SCENE software. The deviation analyses were performed in: (a) the FARO SCENE software with deploying a plug-in application provided by a third party, and (b) CloudCompare, an open source software. A manual registration method with the aid of targets was deployed. A total station was used to obtain the coordinate system for the reference targets. The standardised process for TCM using TLS is proposed based on the literature review, empirical data and experience of the field researcher.

\section{PROPOSAL: TCM USING TLS}

In order to measure and visualise/demonstrate geometric variations more effectively, this paper proposes a standard approach for TCM using TLS. The proposed approach has four standard steps to be followed (Figure 1).
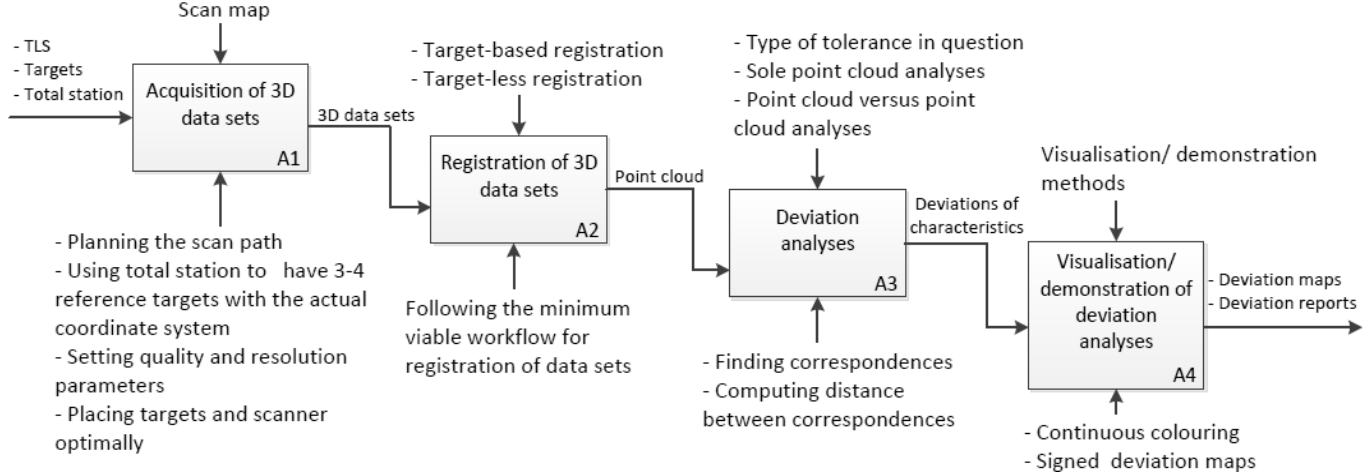

Figure 1: The proposed tolerance compliance measurement using terrestrial laser scanner.

\section{ACQUISITION OF 3D DATA SETS USING TLS}

The first step in the proposed TCM process is to acquire point cloud data sets using TLS. This section recommends the best practice to achieve the highest accuracy. Successful registration of $3 \mathrm{D}$ data sets within the deviation of $3 \mathrm{~mm}$, which is a reasonably high accuracy based on the observed practice in the industry by the field researcher, much depends on the acquisition of 3D data sets.

\section{Scan map}

Planning the scan path in advance is important. The scan map consists of a floor plan that demonstrates the scan positions with the corresponding scan numbers in the field. The scan map is useful to help plan the scan path in an optimum pattern (i.e. zig-zag pattern) 
and optimum distances between scan positions. In other words, using this method will help the operator realise which scans have overlapping and common data, and accordingly which scans should be grouped into a cluster tree. Not planning the scan path proactively using a scan map may result in illogical scan positions, deficient registration, and eventually inaccurate as-built data sets. Figure 2 shows an example of a scan map.

\section{Reference targets}

In the proposed TCM process, it is essential to have a coordinate system in the as-built data set to ensure that the deviation analyses will be performed on a correctly levelled and sized data set. To apply the coordinate system, minimally three and preferably four reference targets (i.e. black and white checkerboard or sphere) should be used. First, the position of targets should be marked on the scan map: (a) to ensure that there is a triangulation for the targets because only this geometry leads to levelled and sized asbuilt data sets, and (b) to demonstrate that at least one scan position will capture the targets. The yellow marks in Figure 2 show the position of four targets in the scan map. The targets then should be placed in the field. A total station should be used to have those targets set up with an actual coordinate system. Having the coordinate system associated with the targets, the as-built data set can be aligned and rotated to a correct size according to the reference coordinate system.

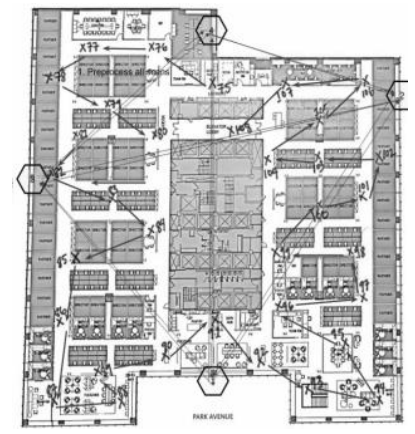

Figure 2: An example of a scan map demonstrating the scan path (blue lines), position of scans (red numbers), and position of the reference targets (yellow marks).

\section{Quality and resolution parameters in TLS}

The basic parameters of quality and resolution should be correctly selected to obtain an accurate as-built data set. Having higher resolution and quality can increase the accuracy of data sets but they also significantly increase the time required to capture data. It is recommended to have more scans at lower scanner settings, rather than having a lower number of scans with higher settings. Hence, an operator should hold a trade-off between the level of resolution and quality needed, and the time constraints to scan a space. To do so, relatively appropriate values for these settings based on the best practice observed and experience gained are suggested in Table 2.

\section{Scanner and targets placement}

Targets are needed only if the Target-based Registration is used. The distance between scan positions should not exceed 10-15 m. A minimum of three targets per scan should be deployed. Also, there should be at least three common targets between scans for 
redundancy purposes. Targets should not be placed in line; instead they should be spread across, and there should be variation in their vertical elevation. The scan positions should not be more than $15 \mathrm{~m}$ away from the target otherwise they will not be recognised by the software used in this research as the number of returns reduces. It is essential that the targets not be occluded from scan positions. This can be ensured by following the scan map. The overall scan positions should form a closed loop. This means starting from a point, doing all scans and finishing at the same point.

Table 2: Suggested values for Resolution and Quality in TLS.

\begin{tabular}{ll}
\hline \multicolumn{1}{c}{ Parameters } & \multicolumn{1}{c}{ Settings } \\
\hline Resolution & For large interior areas and exterior areas, a high resolution $(\sim 1 / 4) ;$ for interior spaces, \\
& medium resolution $(\sim 1 / 8)$, and for small interior spaces low resolution $(\sim 1 / 10)$ are suggested. \\
\hline Quality & $\begin{array}{l}\text { This setting depends on the type of the material of which an object or surface is made. For } \\
\text { non-reflective materials (concrete), the quality parameter of } 2 X, \text { and for highly reflective } \\
\text { surfaces and objects (shiny partition walls), a quality parameter of } 3 X \text { or } 4 X \text { are recommended. }\end{array}$ \\
\end{tabular}

\section{REGISTRATION}

There are two main ways of registering acquired data sets when using the FARO SCENE software: Target-based Registration and Target-less Registration. The Target-based registration relies on targets, whereas Target-less Registration relies on vertical planes (e.g., walls, columns). A minimum viable workflow for registration of 3D data sets is proposed in this paper (Figure 3). Following this workflow, the alignment deviation between targets should be less than $3 \mathrm{~mm}$.

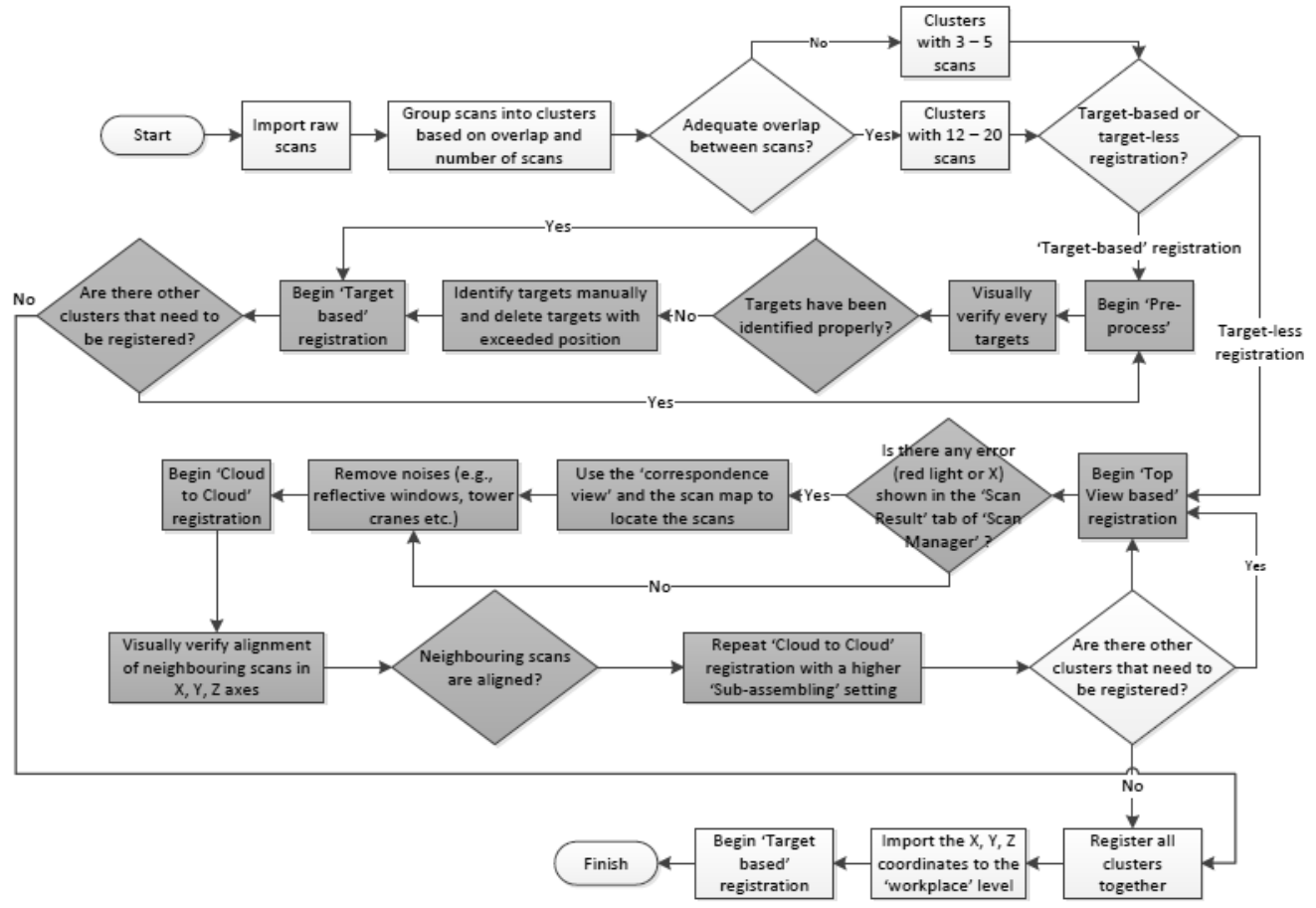

Figure 3: The proposed minimum viable workflow for data registration when using FARO SCENE software 
In the Target-based Registration, pre-processing may be used to identify targets automatically and to apply filters. Targets in every scan should be visually verified to ensure they have been appropriately identified (i.e. there is a sufficient number of returns, targets have been identified only, the position deviation of targets is as low as possible). Eventually, Target-based Registration can be performed. If the operator has taken the recommendations for target placement into account, the desired accuracy is achieved.

In the Target-less Registration, there are two steps: Top View based registration and Cloud to Cloud registration. The former registration is used to align the scans together roughly, and then the latter registration is performed to refine the initial attempt and achieve the desired accuracy. Noises in scans should be removed before running the Cloud to Cloud registration. The deviations shown by the software are just an indication and do not demonstrate the overall deviation between scans; hence, visual verification is needed by checking the registered data set in $\mathrm{Z}$ axis (horizontal surfaces) and $\mathrm{X}, \mathrm{Y}$ axes (vertical surfaces). In the next iteration of the Cloud to Cloud registration, Maximum Search Distance should be larger than mean scan point tension. The Cloud to Cloud registration is repeated until the mean scan point tension is lower than $3 \mathrm{~mm}$.

Table 3: Categories of approaches for deviation analyses, algorithms to compute deviations, and the most suitable algorithms for each type of tolerances

\begin{tabular}{|c|c|}
\hline $\begin{array}{l}\text { Approaches } \\
\text { used for } \\
\text { deviation } \\
\text { analyses }\end{array}$ & Algorithms to compute deviations \\
\hline \multirow[t]{2}{*}{$\begin{array}{l}\text { Sole point cloud } \\
\text { analyses }\end{array}$} & $\begin{array}{l}\text { Point to reference plane: A reference } \\
\text { plane is established for a group of } \\
\text { points in an as-built data set. The } \\
\text { distance between the points in the data } \\
\text { set and the plane is computed. }\end{array}$ \\
\hline & $\begin{array}{l}\text { Point to reference line: A line of best } \\
\text { fit, termed the reference line, is } \\
\text { established and the distance between } \\
\text { the points in the data set and the line is } \\
\text { computed. }\end{array}$ \\
\hline $\begin{array}{l}\text { Point cloud } \\
\text { versus point } \\
\text { cloud analyses }\end{array}$ & $\begin{array}{l}\text { Selected points to selected points: The } \\
\text { shortest point to point distance is } \\
\text { calculated by computing the shortest } \\
\text { Euclidean distance between a point } \\
\text { given in the first point cloud to a } \\
\text { corresponding point in the second point } \\
\text { cloud. The only way to ensure that the } \\
\text { points with similar coordinates from } \\
\text { different scans are selected is to align, }\end{array}$ \\
\hline
\end{tabular}

Flatness and perpendicularity (surface): It is a regular practice to assess flatness of surfaces (e.g., concrete slabs) and perpendicularity of vertical planes (e.g., walls) by using point to reference plane analysis.

Straightness: Deflection in beams can be measured by defining a reference line across the bottom of a beam and measure the distance between points and the best fit line. Hence, point to reference line should be used if straightness in a beam is controlled.

Flatness and straightness: This algorithm can be used to calculate the deviations in a selected grid of points on concrete slabs over time (e.g., post-pour, after tensioning of PT)

Straightness: Changes in camber of beams can be detected by calculating the deviations in manually selected points on 
and size scans according to the reference targets and then select the points with the same coordinates. Otherwise, selected point to selected point comparison will have an error as it is not possible to set the scanner at the same position and select the same points.

Reference points to corresponding points: A reference plane is established in the first scan. The Euclidean distance between a point in the reference plane and the nearest neighbour point in the second point cloud is computed. the bottom of beams.

Flatness, Straightness, Perpendicularity, Profile, Position: This algorithm will help control the deformation in beams and columns (Straightness), changes in the plumbness of any component (Perpendicularity), changes in the level of surfaces (Profile), and changes in the location of components (not distance and coaxiality between components)

(Position) over time.

\section{DEVIATION ANALYSES}

In this paper, deviation analyses based on the data acquired from TLS are divided into two categories, namely: (a) sole point cloud analyses, and (b) point cloud versus point cloud analyses. These analyses have distinct algorithms to compute deviations, they employ a different number of data sets, and each analysis should be used to quantify a specific type of variation (Table 3). It was concluded that Perpendicularity (axis) (e.g., plumbness of columns), Parallelism and Profile cannot be automatically controlled using the described algorithms, although changes in them over time can be detected.

\section{VISUALISATION OF RESULTS OF THE DEVIATION ANALYSES}

Eventually, the deviations are visualised through deviation maps or are demonstrated numerically to reveal deviation patterns. The deviation maps can be produced in the form of heat map (continuous and signed map) (Figure 4a) and contour map. To demonstrate the results of: (a) deformation analysis for beams (Figure 4c), (b) change detection of beams (Figure $4 \mathrm{~b}$ ) and concrete slabs over time, the results are revealed numerically.

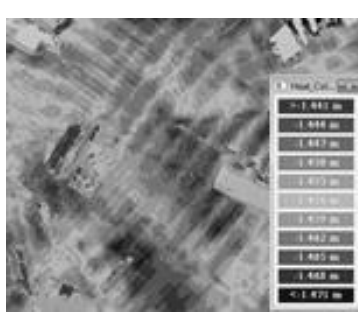

(a)

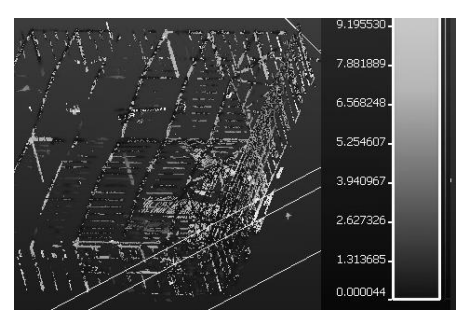

(b)

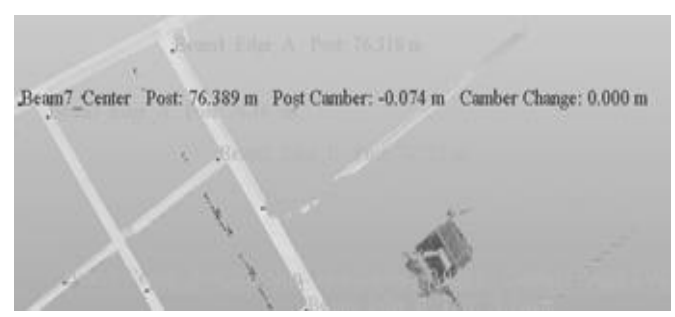

(c)

Figure 4: (a) Heat map for concrete flatness, (b) deviation map for changes in all types of variations over time, and (c) numerical demonstration of deformations in beam over time. 


\section{CONCLUSIONS}

This paper is the first attempt to document a formal and holistic method termed TCM using TLS according to the literature, the best practice observed, and the field researcher's experience. This method has four steps: acquisition of 3D data sets, registration of data sets, deviation analyses, and visualisation/demonstration of deviation analyses. The critical practical recommendations to acquire data and to facilitate data registration were provided. The recommendations include preparation of scan map, placement of scanner and targets, and configuration of scanner settings. A minimum viable workflow for data registration, including both Target-based and Target-less Registration, was suggested. Following the workflow, the operator will be able to achieve less than $3 \mathrm{~mm}$ alignment deviation consistently. The most common approaches and algorithms used in the commercially available software for deviation analyses were categorised appropriately. Different geometric deviations were correlated with different algorithms of deviation analyses. It is envisioned that distinguishing between the types of tolerances facilitates selection of an optimal algorithm for deviation analyses. The results show that the algorithms for deviation analyses do not automatically measure all types of tolerances. One immediate future research will be to investigate how Building Information Modelling and TLS together can quantify each type of geometric variation.

\section{REFERENCES}

Akinci, B., Boukamp, F., Gordon, C., Huber, D., Lyons, C., and Park, K. (2006). "A formalism for utilization of sensor systems and integrated project models for active construction quality control." Automation in construction, 15(2), 124-138.

Anil, E. B., Tang, P., Akinci, B., and Huber, D. (2011). "Assessment of the quality of asis building information models generated from point clouds using deviation analysis." Three-Dimensional Imaging, Interaction, and Measurement, International Society for Optics and Photonics, 78640F.

Anil, E. B., Tang, P., Akinci, B., and Huber, D. (2013). "Deviation analysis method for the assessment of the quality of the as-is Building Information Models generated from point cloud data." Automation in Construction, 35, 507-516.

Bosché, F., and Guenet, E. (2014). "Automating surface flatness control using terrestrial laser scanning and building information models." Automation in construction, 44, 212-226.

Forcada, N., Macarulla, M., Gangolells, M., and Casals, M. (2016). "Handover defects: comparison of construction and post-handover housing defects." Building Research \& Information, 44(3), 279-288.

Holst, C., and Kuhlmann, H. (2016). "Challenges and present fields of action at laser scanner based deformation analyses." Journal of applied geodesy, 10(1), 17-25.

Kim, M.-K., Sohn, H., and Chang, C.-C. (2014). "Automated dimensional quality assessment of precast concrete panels using terrestrial laser scanning." Automation in Construction, 45, 163-177.

Landin, A. (2010). "Demands on the tolerances when industrialising the construction sector." New Perspective in Industrialisation in Construction, 197-205. 
Liker, J. K. (2004). The Toyota way - 14 management principles from the world's greatest manufacturer, McGraw-Hill, New York.

Milberg, C., and Tommelein, I. D. (2005). "Application of tolerance mapping in AEC systems." Proceedings of Construction Research Congress, ASCE, California, 1-10.

Nahangi, M., and Haas, C. T. (2014). "Automated 3D compliance checking in pipe spool fabrication." Advanced Engineering Informatics, 28(4), 360-369.

Olsen, M. J., Kuester, F., Chang, B. J., and Hutchinson, T. C. (2009). "Terrestrial laser scanning-based structural damage assessment." Journal of Computing in Civil Engineering, 24(3), 264-272.

Phares, B. M., Washer, G. A., Rolander, D. D., Graybeal, B. A., and Moore, M. (2004). "Routine highway bridge inspection condition documentation accuracy and reliability." Journal of Bridge Engineering, 9(4), 403-413.

Talebi, S., Koskela, L., Tzortzopoulos, P., and Krulikowski, A. (submitted). "Deploying Geometric Dimensioning and Tolerancing in Construction." Manuscript submitted for publication.

Tang, P., Anil, E. B., Akinci, B., and Huber, D. (2011). "Efficient and effective quality assessment of as-is building information models and 3D laser-scanned data." Computing in Civil Engineering, 486-493.

Tang, P., Huber, D., and Akinci, B. (2010). "Characterization of laser scanners and algorithms for detecting flatness defects on concrete surfaces." Journal of Computing in Civil Engineering, 25(1), 31-42.

Wilkes, P., Lau, A., Disney, M., Calders, K., Burt, A., de Tanago, J. G., Bartholomeus, H., Brede, B., and Herold, M. (2017). "Data acquisition considerations for Terrestrial Laser Scanning of forest plots." Remote Sensing of Environment, 196, 140-153.

Womack, J. P., and Jones, D. T. (1997). "Lean thinking-banish waste and create wealth in your corporation." Journal of the Operational Research Society, 48(11), 1148-1148.

Yates, J. K., and Lockley, E. E. (2002). "Documenting and analyzing construction failures." Journal of construction Engineering and management, 128(1), 8-17. 